\title{
DESENVOLVIMENTO DE METODOLOGIA PARA AVALIAÇÃO DE AÇOS ESFEROIDIZADOS. PARTE 2: DETERMINAÇÃO DO GRAU DE ESFEROIDIZAÇÃO
}

\author{
Pedro Carlos Hernandez Júnior ${ }^{1}$ \\ José Eduardo Ferreira da Fonseca ${ }^{2}$ \\ Luís Frederico Pinheiro Dick ${ }^{3}$
}

\section{Resumo}

Desenvolveu-se neste trabalho metodologia simples para avaliar quantitativamente a microestrutura de aços esferoidizados. Na primeira parte, analisou-se a descarbonetação destes aços e, no presente trabalho, o seu grau de esferoidização, tendo em vista que as normas existentes se limitam ao uso de padrões qualitativos para determinar o grau de esferoidização médio. Estudou-se o aço AISI 1095 na forma de fio-máquina esferoidizado. Para isto, utilizou-se o parâmetro circularidade em micrografias eletrônicas de varredura, possibilitando quantificar a distribuição de carbonetos conforme seu grau de esferoidização.

Palavras-chave: Esferoidização; Micrografia; Análise de imagem; Aço SAE 1095.

\section{DEVELOPMENT OF METHODOLOGY FOR THE EVALUATION OF SPHEROIDIZED STEELS. PART 2: DETERMINATION OF THE SPHEROIDIZATION DEGREE}

\begin{abstract} quantification of the carbides distribution according to their spheroidization degree.

Key words: Spheroidization; Micrographs; Image analysis; SAE I095 Steel.

\section{INTRODUÇÃO}

O tratamento térmico de esferoidização é realizado nos aços perlíticos com o objetivo de aumentar a sua ductilidade. Essa condição é atingida com a transformação de carbonetos lamelares, formados sob velocidades baixas de resfriamento, em carbonetos aproximadamente esféricos em matriz ferrítica. ${ }^{(1)}$

Normalmente, na produção de aços de alto carbono, o tratamento térmico de esferoidização é realizado após a laminação ou o forjamento, nos chamados semiprodutos, exigindo um grau de esferoidização específico para seu processamento posterior, por exemplo, de $80 \%$ a $90 \%$ de carbonetos globulares. ${ }^{(2)}$
\end{abstract}

In this work, a simple methodology was developed for the quantitative evaluation of the microstructure of spheroidized steels. In the first part, the decarburization of these steels was analyzed and now, methodology for the determination of the spheroidization degree was developed, as the available industrial norms are restricted to the use of qualitative standards for determining the mean spheroidization degree. The SAE 1095 steel was studied in the spheroidized annealed condition. The parameter circularity was applied to scanning electron micrographs, enabling the

A esferoidização de carbonetos ocorre via difusão do carbono intersticial na rede e difusão superficial na interface carboneto/matriz em um processo de coalescimento. A força motriz é a diminuição da energia superficial pela mudança da forma dos carbonetos lamelares para globulares e, posteriormente, pelo seu crescimento. É muito difícil diferenciar os estágios de esferoidização por análises qualitativas pelas possíveis irregularidades presentes na estrutura da cementita lamelar. Estudos quantitativos da razão de

'Prof. MSc. do IFET Sul-Riograndense e Doutorando do Programa de Pós-Graduação em Engenharia de Minas, Metalúrgica e de Materiais, Universidade Federal do Rio Grande do Sul - UFRGS, Av. Copacabana, 100, CEP 932 16-I20, Sapucaia do Sul, RS, Brasil. E-mail: pedro.hernandez@ufrgs.br ${ }^{2}$ Graduando Engenharia Metalúrgica, Departamento de Engenharia Metalúrgica, Universidade Federal do Rio Grande do Sul - UFRGS,

Av. Bento Gonçalves, 9500, Bloco 4, Prédio 75, $2^{\circ}$ Andar, CEP 9150I-970, Porto Alegre, RS, Brasil. E-mail: gt_jeff@hotmail.com

${ }^{3}$ Prof. Dr., Departamento de Metalurgia e Centro de Microscopia Eletrônica, Universidade Federal do Rio Grande do Sul - UFRGS,

Av. Bento Gonçalves, 9500, Bloco 4, Prédio 75, 2ºndar, CEP9150I-970, Porto Alegre, RS, Brasil. E-mail: Ifdick@ufrgs.br 
forma (aspect ratio) da cementita, definida como o comprimento dividido pela largura das lamelas, são necessários para evidenciar os diferentes estágios de esferoidização e o fim do tratamento térmico.

A sequência de eventos envolvida é assim descrita: ${ }^{(3)}$

- As placas de cementita se rompem em intervalos mais ou menos regulares, pelo surgimento de canais nas placas, até que sua razão de forma atinja um valor ao redor de 8;

- O valor da razão de forma dos fragmentos das placas se reduz progressivamente para I, gerando formas aproximadamente esféricas; e

- O diâmetro médio das esferas aumenta pela eliminação das esferas menores com o consequente crescimento das maiores pelo processo conhecido por Ostwald ripening.

Os principais processos de tratamento térmico utilizados para a obtenção desta estrutura são:( ${ }^{(1)}$

- Manutenção prolongada em temperaturas logo abaixo da temperatura eutetóide (linha $\mathrm{A}_{1}$ do diagrama Fe-C);

- Aquecimento e resfriamento alternado entre temperaturas acima e abaixo de $\mathrm{A}_{1}$;

- Aquecimento a temperaturas acima de $A_{1}$ seguido de resfriamento lento no forno; ou

- Manutenção da temperatura logo abaixo de $A_{1}$ e posterior resfriamento lento.

Como a esferoidização exige um tempo longo em altas temperaturas, a possibilidade de ocorrer descarbonetação é na prática um problema frequente. As normas para sua caracterização são aplicáveis apenas a estruturas perlíticas. Por isto, em trabalho anterior, ${ }^{(4)}$ foi desenvolvida metodologia baseada na análise de imagens de microscopia eletrônica de varredura (MEV) para quantificação da descarbonetação.

Nas principais normas existentes para a medição do grau de esferoidização $(\mathrm{GE})^{(5,6)}$, as frações volumétricas de glóbulos de carbonetos são determinadas apenas qualitativamente. Neste trabalho, visando suprir esta lacuna, objetivou-se desenvolver um método analítico quantitativo preciso para determinar o Grau de Esferoidização com a utilização das técnicas de microscopia eletrônica de varredura, microscopia óptica e software analisador de imagens.

\section{MATERIAIS E MÉTODOS}

Foram analisadas amostras de fio-máquina de aço SAE 1095, com diâmetro de 7,94 mm, cortadas de espira de 1,0 m de diâmetro. A análise química do aço é mostrada na Tabela I. Um efetivo contraste do perímetro dos carbonetos para posterior análise de imagens de MEV foi obtido com a utilização de curto ataque por $10 \mathrm{~s}$ com reagente Nital $2 \%$.

Tabela I. Composição química das amostras de aço SAE 1095.

\begin{tabular}{ccccccccc}
\hline$\% \mathrm{C}$ & $\% \mathrm{Si}$ & $\% \mathrm{Mn}$ & $\% \mathrm{P}$ & $\% \mathrm{~S}$ & $\% \mathrm{Cr}$ & $\% \mathrm{Ni}$ & $\% \mathrm{Mo}$ & $\% \mathrm{Cu}$ \\
\hline 0,98 & 0,21 & 0,39 & 0,02 & 0,01 & 0,06 & 0,06 & 0,02 & 0,09 \\
\hline
\end{tabular}

As amostras de espiras de fio-máquina foram cortadas em barras de cerca de 15,0 cm e preparadas para a análise metalográfica por procedimento descrito anteriormente. ${ }^{(4)}$

Para a realização das análises de imagem quantitativas foi utilizado o software de utilização livre Image J v. I.38x. As imagens de cada amostra esferoidizada foram tratadas de forma a evidenciar os carbonetos presentes e eliminar artefatos que adicionariam erro às medidas. Após identificação das partículas por ajuste de contraste, foram determinados o perímetro e a área de cada partícula para uso posterior.

\section{RESULTADOS E DISCUSSÃO}

Assim como para o grau de descarbonetação, ${ }^{(4)}$ com a utilização de métodos estereológicos pode-se também propor uma forma quantitativa para medir o GE, havendo neste caso dois pontos principais: (i) a forma de cada partícula e (ii) a distribuição de partículas conforme esta forma. A razão de forma RF (largura/altura) pode ser usada para isto, tomando-se o máximo e o mínimo diâmetros da seção de cada carboneto. No entanto, para estruturas lamelares finas com superfícies curvas, a determinação automática por análise de imagens dos diâmetros máximos e mínimos não produziu resultados satisfatórios, principalmente para estruturas pouco esferoidizadas ou irregulares. Tendo em vista que a área em pixels e o perímetro são de fácil determinação automática por análise de imagens, utilizou-se o parâmetro circularidade, expresso a partir da área A e do perímetro P:

$$
\text { Circularidade }=\frac{4 \pi \mathrm{A}}{\mathrm{P}^{2}}
$$

Observa-se que, quanto mais a forma de uma partícula se aproxima de um círculo, mais o valor da sua circularidade se aproxima de I, como indica a Figura I. Comparando-se a razão de forma e circularidade de diferentes figuras, verifica-se que, para elipses e retângulos, RF é a mesma, caso o eixo maior e o menor sejam iguais, enquanto que a circularidade $\mathrm{C}$ difere.
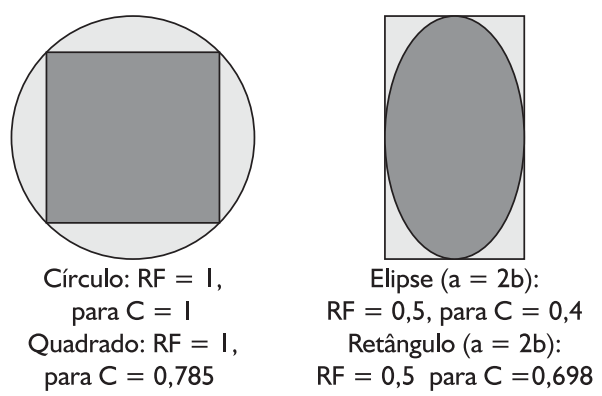

Figura I. Relações entre RF e C para formas retangulares e elípticas. 
método utilizado é desenvolvido a partir da obtenção de micrografias com bom contraste, seguido da binarização das imagens com o objetivo de possibilitar a contagem e a medição das mesmas.

A Figura 2 apresenta a vista ao MEV do núcleo do fio-máquina SAE 1095 de 7,94 mm de diâmetro com aumento de $10.000 \times$ e, a Figura 3, o histograma de distribuição de valores de circularidade dos carbonetos. Arbitrando-se como critério para esferoidização uma circularidade mínima de 0,5, a quantidade de partículas que atendem a esta condição é de cerca de $80 \%$ (Figura 3).

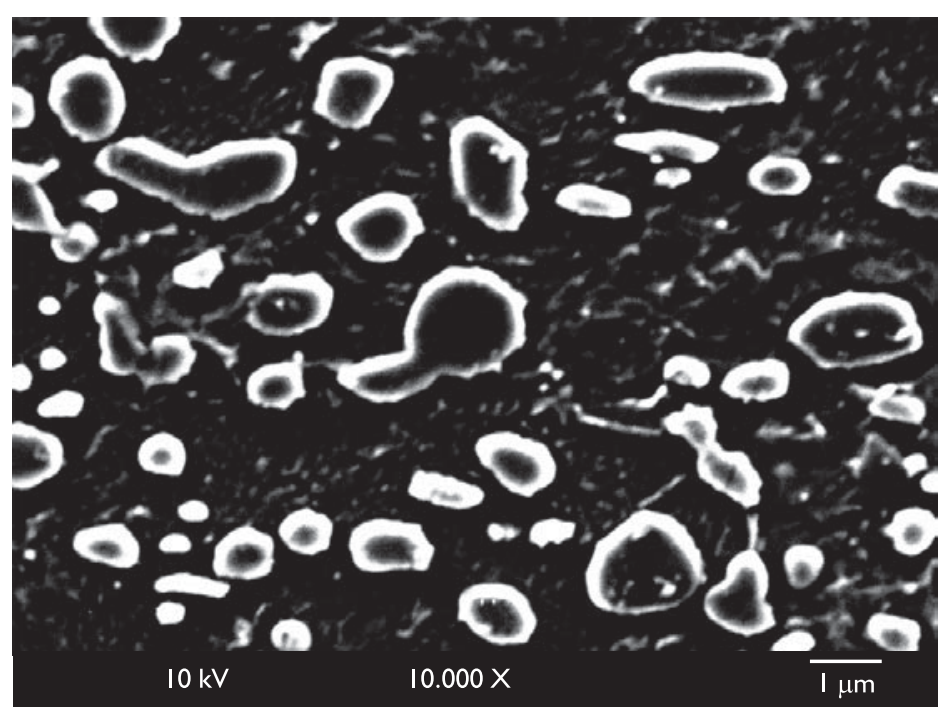

Figura 2. Núcleo do fio-máquina de 7,94 mm. MEV: 10.000×.

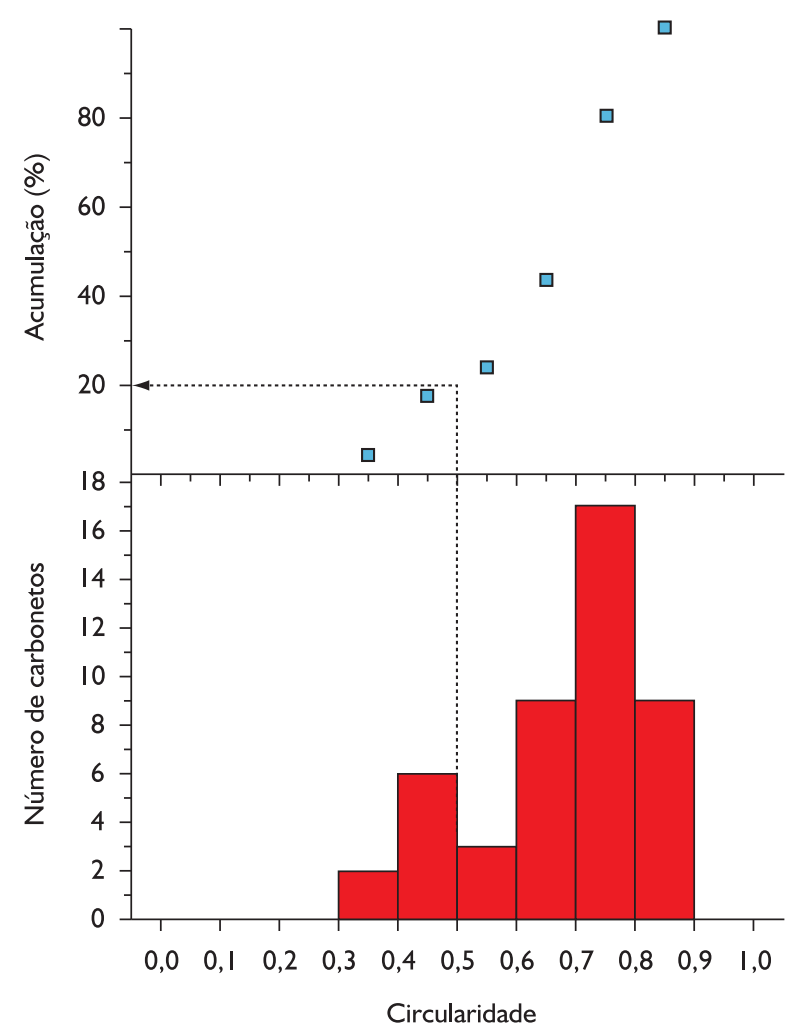

Figura 3. Histograma de distribuição da circularidade das partículas da micrografia da Figura 2 com $10.000 \times$.
A utilização de diferentes aumentos acarreta diferentes áreas de amostragem. Portanto, são apresentados, também, exemplos de aplicação do método para diferentes magnificações de micrografias da mesma amostra.

$\mathrm{Na}$ Figura 4 é apresentada a micrografia de núcleo de aço SAE 1095 de $7,94 \mathrm{~mm}$ com aumento de $5.000 \times$. Na Figura 5 é apresentado o histograma de distribuição de circularidade dos carbonetos. Verifica-se que, se for utilizado o mesmo critério do exemplo anterior, a quantidade de partículas que atende à condição especificada, para este aumento $(5.000 \times)$ fica em torno de $50 \%$. Observa-se, portanto, uma variação dos valores para a mesma amostra, dependendo do aumento utilizado.

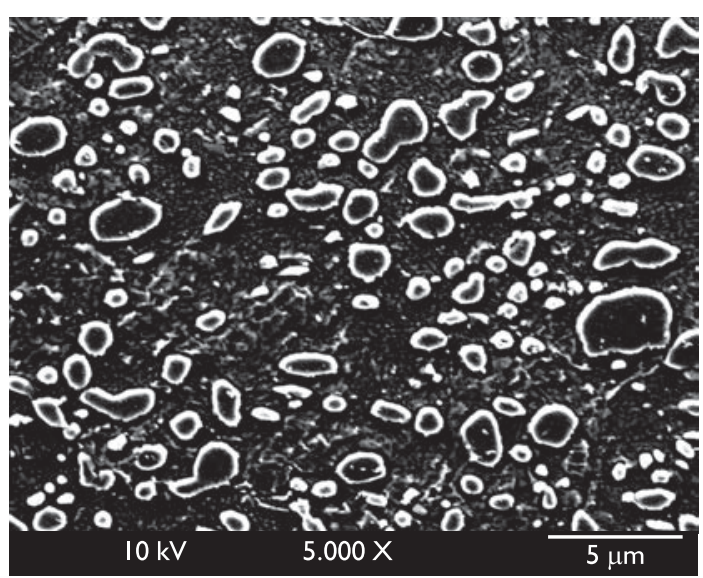

Figura 4. Núcleo do fio-máquina de 7,94 mm. MEV: 5.000×.

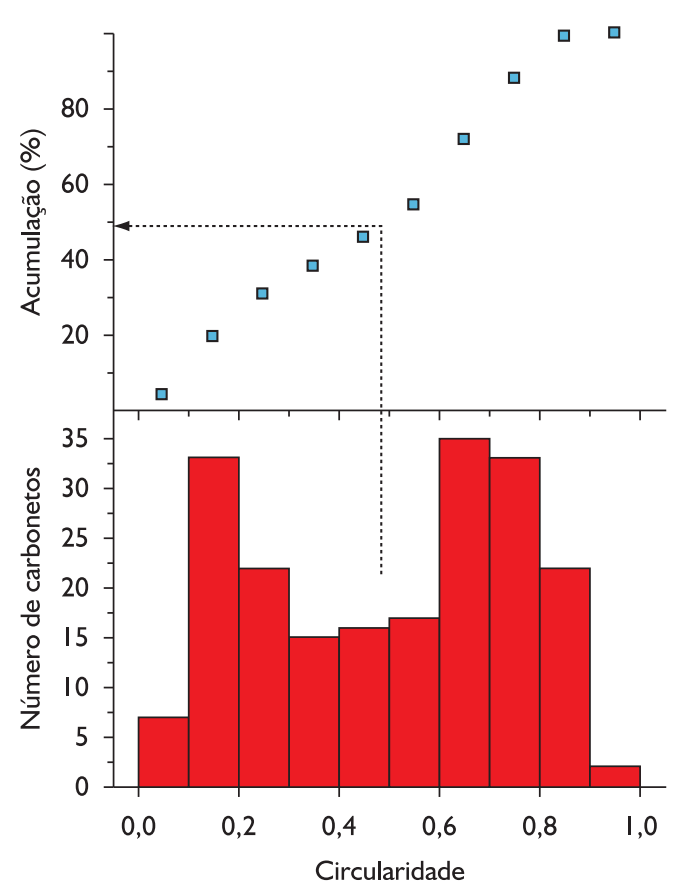

Figura 5. Histograma da distribuição da circularidade das partículas da micrografia da Figura 4 com $5.000 \times$. 


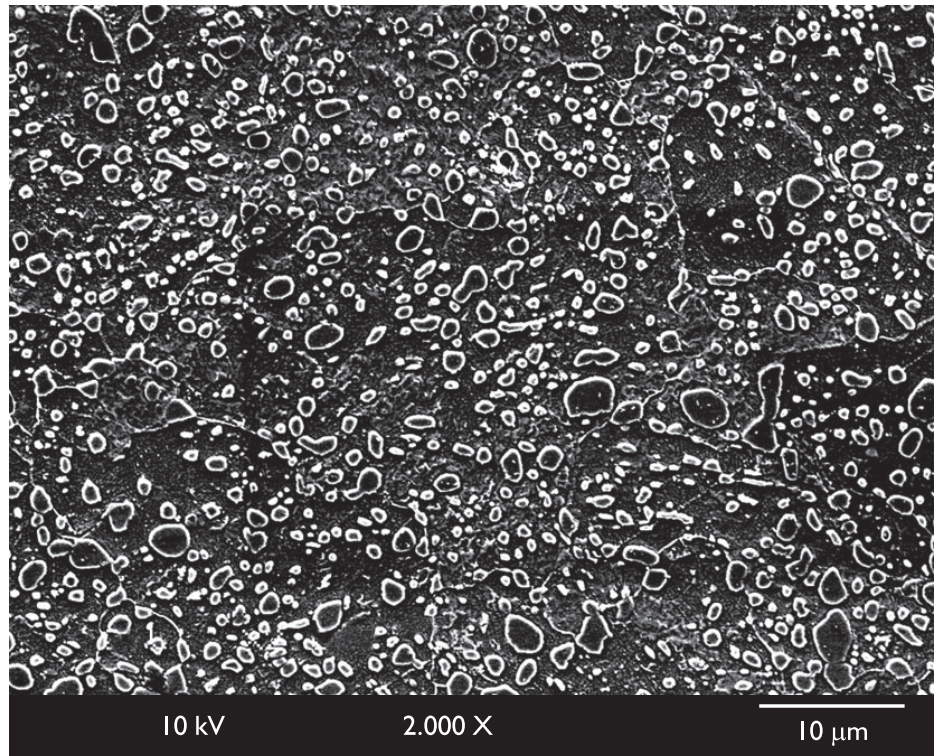

Figura 6. Núcleo do fio-máquina de 7,94 mm. Micrografia original: $2.000 \times$.

Análises feitas na mesma amostra (Figuras 6 e 7), porém com aumento menor $(2.000 \times)$ e consequente área maior de amostragem, apresentaram resultado diverso do da imagem feita com aumento de $5.000 \times$, com a quantidade de partículas que atendem a condição especificada em torno de $70 \%$. Tal resultado pode ser explicado pela melhor estatística da imagem com aumento de $2.000 \times$, com maior quantidade de partículas e maior área.

Assim, como para a determinação do grau de descarbonetação, ${ }^{(4)}$ é possível também obter diferentes parâmetros a partir dos perfis obtidos com a análise de dados com uma resolução superior à da técnica de microscopia óptica feita por comparação com padrões micrográficos. Trata-se de uma poderosa ferramenta para quantificar o grau de esferoidização.

\section{CONCLUSÕES}

O método desenvolvido mostra que é possível a quantificação do grau de esferoidização com resolução espacial na profundidade de cerca de 5,0 $\mu \mathrm{m}$, minimizando as variações associadas aos métodos convencionais por comparação com padrões micrográficos.

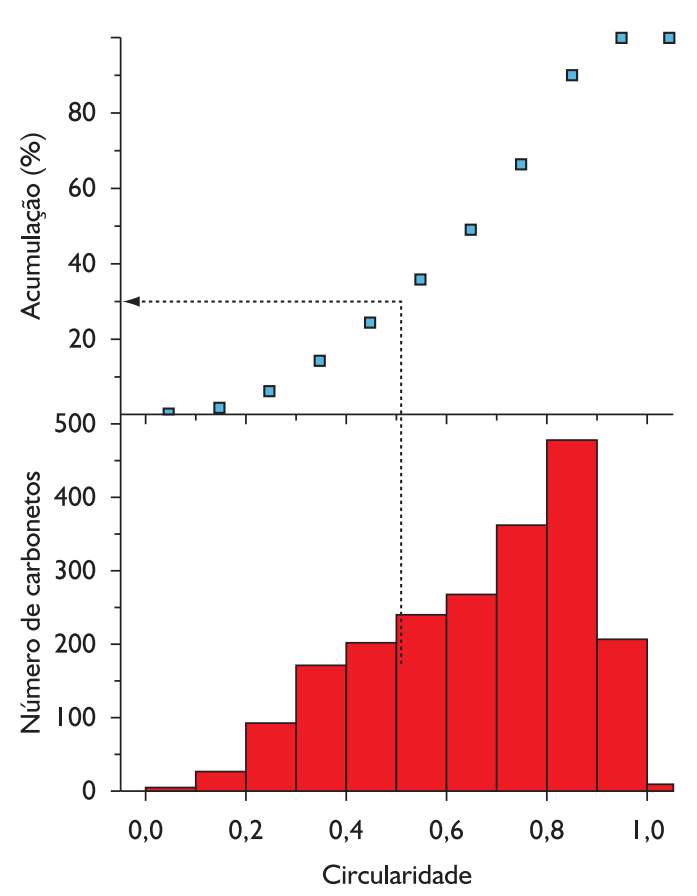

Figura 7. Histograma de distribuição da circularidade das partículas do núcleo do fio-máquina, referente à micrografia da Figura 6.

Para a análise de microestruturas esferoidizadas, como a do fio-máquina de $6,35 \mathrm{~mm}$ de diâmetro do exemplo, a determinação do grau de esferoidização necessita de imagens com aumento igual ou inferior a $2.000 \times$.

A mesma metodologia pode ser utilizada também para a medição do grau de descarbonetação.

\section{Agradecimentos}

Os autores agradecem ao Centro de Microscopia Eletrônica da UFRGS pelo uso de equipamentos.

\section{REFERÊNCIAS}

I BRAMFITT, B. L.; HINGWE, A. K. Annealing of steel. In: AMERICAN SOCIETY OF METALS. Metals Handbook. 9. ed. Metals Park, Ohio: ASM, 1991. v. 4: Heat treatment, p. 42-55.

2 TOTTEN, G. E. Steel heat treatment, metallurgy and technologies. 2. ed. Boca Raton: CRC Press, 2007.

3 SAMUELS, L. E. Light microscopy of carbon steels. Metals Park, Ohio: ASM, 1999.

4 HERNANDEZ JUNIOR, P. C.; FONSECA, J. E. F.; DICK, L. F. P. Desenvolvimento de metodologia para avaliação de aços esferoidizados. Parte I: determinação do grau de descarbonetação. Tecnologia em Metalurgia e Materiais, 2010.

5 ASSOCIAÇÃO BRASILEIRA DE NORMAS TÉCNICAS. ABNT NBR I 4677 - Aço: determinação do grau de esferoidização de carbonetos: método de ensaio. Rio de Janeiro, $200 \mathrm{I}$.

6 STAHL-EISEN-PRUFBLÄTTER DES VEREINS DEUTSCHER EISENHUTTENLEUTE. Norma SEP 1520 - Microscopic examination of carbide structure in steels by means of diagram series. 3. ed. Dusseldorf, 1998.

Recebido em: 9/10/2009

Aceito em: 4/03/2010 